\title{
Modeling of Node Energy Consumption for Wireless Sensor Networks
}

\author{
Hai-Ying Zhou, Dan-Yan Luo, Yan Gao, De-Cheng Zuo \\ School of Computer Science and Technology, Harbin Institute of Technology, Harbin, China \\ E-mail: \{haiyingzhou, zuodc\}@hit.edu.cn, \{luody, gaoyan\}@ftcl.hit.edu.cn \\ Received January 4, 2011; revised January 7, 2011; accepted January 9, 2011
}

\begin{abstract}
Energy consumption is the core issue in wireless sensor networks (WSN). To generate a node energy model that can accurately reveal the energy consumption of sensor nodes is an extremely important part of protocol development, system design and performance evaluation in WSNs. In this paper, by studying component energy consumption in different node states and within state transitions, the authors present the energy models of the node core components, including processors, RF modules and sensors. Furthermore, this paper reveals the energy correlations between node components, and then establishes the node energy model based on the event-trigger mechanism. Finally, the authors simulate the energy models of node components and then evaluate the energy consumption of network protocols based on this node energy model. The proposed model can be used to analyze the WSNs energy consumption, to evaluate communication protocols, to deploy nodes and then to construct WSN applications.
\end{abstract}

Keywords: Wireless Sensor Networks, Energy Model, Event-Trigger

\section{Introduction}

Currently, researches on the basic theories and system models of WSN (Wireless Sensor Networks) are not perfect, especially due to lack of a set of WSN models that can accurately reveal WSN characteristics [1]. Traditional sensor networks address the system QoS, in which the node energy supply is unlimited and the energy issue is not the main problem for its application [2]. In WSN applications, in views of limited power resource (batteries) and long lifetime requirement, the energy consumption becomes the core issue in WSN designs. Most of studies focus on the energy efficiency and optimization techniques for the design of WSN platforms, system software and protocols. Some WSN system models and simulation technologies have been studied, but these studies generally focused on the development of simulation platforms and modeling of communication protocols, being lack of the basic system model for platforms and protocols. Some typical simulation tools and platforms, such as OPENET, NS2, SHAWN, SensorSim, EmStar, OMNet, GloMoSim, TOSSIM, PowerTOSSIM etc., are implemented basing on the traditional mathematical models of wireless network, which can not accurately reflect the features of WSN applications, resulting in low precision simulation. In addition, current studies are lack of modeling of WSN energy consumption, and thus cannot evaluate the key performance indicator of WSN-system lifetime. Traditional energy modeling method is to deduce the node energy consumption by analyzing and modeling the energy consumption of each node module based on the theoretical energy consumption data or model of different modules. This method cannot analyze and evaluate the node energy situations from the views of the energy supply module (battery) and the energy consumption modules, i.e., processor module (PM), transceiver module (TM) and sensor module (SM), in different operation mode [3].

WSN applications can provide the unattended and long-time surveillance services, which implements the basic function of signal collection, processing and transmission. Due to low accuracy caused by error or inaccurate system models, current WSN simulation platforms (tools) have seriously affected the protocol development, system construction and performance analysis of WSN system, and thus impeded the WSN industrialization. This paper addresses the research on the WSN energy modeling, aiming to provide the accurate energy model of WSN node to improve the simulation accuracy. 


\section{Node Energy Modeling}

WSN nodes consist of several functional modules: Microprocessor, Transceiver, Sensor, and power supply modules. By studying the energy consumption issues of node components in different component states and during state transitions, this section presents the energy models of the processor module, communication module and sensing module of WSN nodes, and then establishes the node energy model by adopting the event-driven mechanism.

\subsection{Processor Energy Model (PEM)}

1) Processor Operation State: The processor module is the node control and data processing center, being responsible for sensor controlling, protocol communicating and data processing, and etc. The microprocessor normally supports three operation states (sleep, idle, run) and has five state transitions [4], shown in Figure 1:

2) Processor Energy Function: Processor energy consumption $\left(E_{c p u}\right)$ is the sum of the state energy consumption $\left(E_{\text {cpu-state }}\right)$ and the state-transition energy consumption $\left(E_{\text {cpu-change }}\right)$, as in (1), while $i=1,2, \cdots, m$, $i$ is the processor operation state and $m$ is the number of the processor state $(m=3) ; j=1,2, \cdots, n, j$ is the type of state transition and $n$ is the number of the state-transition $(n=5)$.

$$
\begin{aligned}
E_{c p u} & =E_{c p u-s t a t e}+E_{c p u-c h a n g e} \\
& =\sum_{i=1}^{m} P_{c p u-s t a t e}(i) T_{c p u-s t a t e}(i)+\sum_{j=1}^{n} N_{c p u-c h a n g e}(j) e_{c p u-c h a n g e}(j)
\end{aligned}
$$

where $P_{\text {cpu-state }}(i)$ is the power of state $i$ that can be found from the reference manual, and $T_{\text {cpu-state }}(i)$ is the time interval in state $i$ which is a statistical variable that can be calculated in this processor energy model. $N_{\text {cpu-change }}(j)$ is the frequency of state transition $j$, and $e_{\text {cpu-change }}(j)$ is the energy consumption of one-time state transition $j$. To simplify the processor energy modeling, $e_{\text {cpu-change }}(j)$ which can be expressed as in (2).

$$
e_{\text {cpu-change }}(j)=T_{\text {init-end }}(j)\left(P_{\text {init }}(j)+P_{\text {end }}(j)\right) / 2
$$

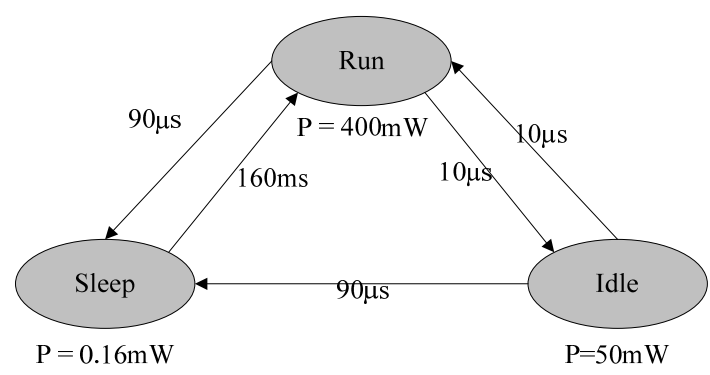

Figure 1. State transition diagram of PM. where $P_{\text {init }}(j)$ and $P_{\text {end }}(j)$ are the power of state init and end in the state transition $j, T_{\text {init-end }}(j)$ is the time interval for the state transition $j$ from the init state to the end state. The power of state transition is considered as the average power of state init and end.

\subsection{Transceiver Energy Model (TEM)}

1) Transceiver Operation State: The communication module includes baseband and RF, being responsible for nodes data sending and receiving. The transceiver normally has six states $\left(T_{x}, R_{x}\right.$, Off, Idle, Sleep, CCA/ED) and nine state transition [5], shown in Figure 2.

2) Transceiver Energy Function: Being similar to the processor energy function, Transceiver energy consumption ( $\left.E_{\text {trans }}\right)$ is the sum of state energy consumption $\left(E_{\text {trans-state }}\right)$ and state-transition energy consumption $\left(E_{\text {trans-change }}\right) . E_{\text {trans-state }}$ can be expressed as in (3).

$$
\begin{aligned}
& E_{\text {trans-state }}=E_{T X}+E_{R X}+E_{\text {Idle }}+E_{\text {sleep }}+E_{C C A} \\
& =\sum_{i=1}^{N_{T X}} P_{T X} L_{i} / R+\sum_{i=1}^{N_{R X}} P_{R X} L_{i} / R+P_{\text {Idle }} T_{\text {Idle }} \\
& \\
& +P_{\text {sleep }} T_{\text {sleep }}+P_{C C A} T_{C C A} \\
& =\sum_{i=1}^{N_{T X}} V_{\text {tr }} I_{T X} L_{i} / R+\sum_{i=1}^{N_{R X}} V_{t r} I_{R X} L_{i} / R \\
& \\
& +V_{\text {tr }}\left(I_{\text {Idle }} T_{\text {Idle }}+I_{\text {sleep }} T_{\text {sleep }}+I_{C C A} T_{C C A}\right)
\end{aligned}
$$

where $E_{x}, P_{x}, I_{x}$, and $T_{x}$ are the energy consumption, the power, the electric current and the time interval of transceiver in state $x . V_{t r}$ is the working voltage, $L_{\mathrm{i}}$ is the size length of the $i^{\text {th }}$ packet of receiving or sending, $R$ is the data transferring rate, $N_{t x}$ and $N_{r x}$ are the total numbers of sending and receiving packets. $E_{\text {trans-change }}$ can be expressed as in (4), where $j=1,2, \cdots, n, j$ is the type of state transition and $n$ is the number of the state-transition $(n=9)$. $N_{\text {trans-change }}(j)$ is the frequency of state transition $j$, and $e_{\text {trans-change }}(j)$ is the energy consumption of one-time state transition $j$, which can expressed as in (5).

$$
\begin{aligned}
E_{\text {trans-transition }} & =\sum_{j=1}^{n} N_{\text {trans-change }}(j) e_{\text {trans-change }}(j) \\
e_{\text {trans-change }}(j) & =T_{\text {init-end }}(j)\left(P_{\text {init }}(j)+P_{\text {end }}(j)\right) / 2 \\
& =V_{\text {tr }} T_{\text {init-end }}(j)\left(I_{\text {init }}(j)+I_{\text {end }}(j)\right) / 2
\end{aligned}
$$

\subsection{Sensor Energy Model (SEM)}

The sensing module consists of sensors and digital-analog converters, be responsible for the information collection and digital conversion. The energy consumption of the sensing module come from multiple operations, including signal sampling, AD signal conversion, and sig- 
nal modulation, etc. The sensing module can operate either in burst or periodic mode.

In this paper, the sensing module is supposed to operate in the periodic mode, in which sensors are opened and closed periodically and then the module enters the 'on' and 'off' states alternately. Supposing the energy consumptions of the 'open' and 'close' operations are constant, the sensor energy consumption $\left(E_{\text {sensor }}\right)$ can be expressed as in (6).

$$
\begin{aligned}
& E_{\text {sensor }}=E_{\text {on-off }}+E_{\text {off -on }}+E_{\text {sensor-run }} \\
& =N\left(e_{\text {on-off }}+e_{\text {off-on }}+V_{s} I_{s} T_{s}\right)
\end{aligned}
$$

where $e_{\text {on-off }}$ is the one time energy consumption of closing sensor operation, $e_{\text {off-on }}$ is the one time energy consumption of opening sensor operation, $E_{\text {sensor-run }}$ is the energy consumption of sensing operation. $V_{s}$ and $I_{s}$ are the working voltage and current of sensors, $T_{s}$ is the time interval of sensing operation, $N$ is the number of sensor opening and closing operation.

\subsection{Nole Energy Model (NEM)}

In real systems, the processor, transceiver and sensor components of WSN nodes must work cooperatively to perform a task, and thus have mutual relationships especially concerning with the energy issue.

The node modules perform tasks and state transitions triggered by different external or internal events. When taking into account the origination of events, they can be classified into two types: events coming from outside and inside of the node energy model. The external events are triggered by external requests or commands, for example, external clock interrupts, sending packet requests, packet arriving action, channel detection commands and etc; the internal events are triggered by internal responses or actions come, such as sending packet action, receiving packet action and periodical data collection. The node overall energy consumption model is shown in

Figure 2, and the event-driven mechanism of different node modules is as follows:

- Event trigger in the sensor module: The sensor energy model (SEM) enters the 'on' state periodically triggered by the external clock event. After sensing and statistically calculating sensor energy consumption, the senor module enters 'off' state automatically.

- Event trigger in the processor module: The processor energy model (PEM) enters the 'run' state triggered by the following three events: the periodical data collection event generated by the sensor energy model; the sending packet requests generated by external applications or protocols; the packet arriving action generated by the transceiver energy

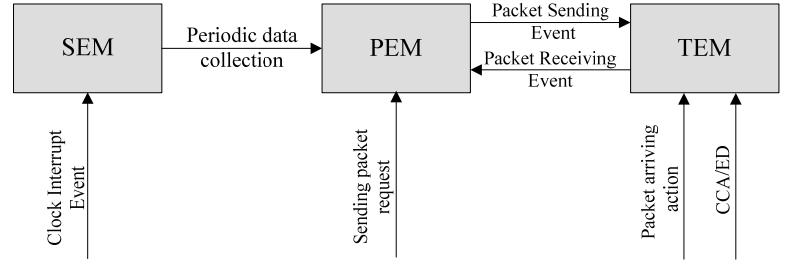

Figure 2. Event trigger mechanism of NEM.

model (TEM).

- Event trigger in the transceiver module: The transceiver energy module (TEM) enters the 'Tx' state triggered by the sending packet event generated by $\mathrm{PEM}$, enters the ' $\mathrm{Rx}$ ' state triggered by the external packet arriving action, and enters the 'CCA/ED' state triggered by channel detection commands.

\section{Simulation of Energy Models}

In order to simulate and evaluate the node energy model in OPENET simulation environment [6], we suppose a WSN node that consists of a Intel Strong ARM SA-1100 Microprocessor [7], a Chipcon CC2420 transceiver [8] and a Dallas digital temperature DS18B20 [9]. Table 1 presents the state power and state transition time of Intel Strong ARM SA-1100 and Table 2 lists the state current and state transition time of Chipcon CC2420. The work voltage $\left(V_{s}\right)$ and current $\left(I_{s}\right)$ of DS18B20 are $5 \mathrm{~V}$ and1mA, and the conversion time is $750 \mathrm{~ms}$. Supposing the switch energy consumption of DS18B20 are $e_{\text {off-on }}=0.0002 \mathrm{~J}$ and $e_{\text {on-off }}=0.0001 \mathrm{~J}$.

In this simulation, the node model adopts the AODV routing protocol [10]. The simulation time length is set to $200 \mathrm{~s}$ : in $0-50 \mathrm{~s}$, data is sent with $1 \mathrm{~s}$ period; in $50-130 \mathrm{~s}$, data is sent by Possion distribution with the expected value of $10 \mathrm{~s}$; after $130 \mathrm{~s}$, data is sent with 1s period. The range of wireless communication is $200 \mathrm{~m}$. The analysis of energy consumption is based on a random selecting node.

Table 1. State power and transition time of Strong ARM SA-1100.

\begin{tabular}{cc}
\hline State & Power(mW) \\
\hline Run & 400 \\
Idle & 50 \\
Sleep & 0.16 \\
State transition Class & State transition time \\
$\mathrm{T}_{\text {run-idle }}$ & $10 \mu \mathrm{s}$ \\
$\mathrm{T}_{\text {idle-run }}$ & $10 \mu \mathrm{s}$ \\
$\mathrm{T}_{\text {idle-sleep }}$ & $90 \mu \mathrm{s}$ \\
$\mathrm{T}_{\text {run-sleep }}$ & $90 \mu \mathrm{s}$ \\
$\mathrm{T}_{\text {sleep-run }}$ & $160 \mathrm{~ms}$ \\
\hline
\end{tabular}


Table 2. State current and transition time of CC2420.

\begin{tabular}{cc}
\hline State & Current \\
\hline $\mathrm{I}_{\text {off }}$ & $0.02 \mu \mathrm{A}$ \\
$\mathrm{I}_{\mathrm{tx}}$ & $17.4 \mathrm{~mA}$ \\
$\mathrm{I}_{\mathrm{Ix}}$ & $19.7 \mathrm{~mA}$ \\
$\mathrm{I}_{\text {idle }}$ & $426 \mu \mathrm{A}$ \\
$\mathrm{I}_{\text {cca/ed }}$ & $17.4 \mathrm{~mA}$ \\
$\mathrm{I}_{\text {sleep }}$ & $20 \mu \mathrm{A}$ \\
State transition Class & State transition time \\
$\mathrm{T}_{\text {off-idle }}$ & $1 \mathrm{~ms}$ \\
$\mathrm{~T}_{\text {cca/ed-idle }}$ & $2 \mu \mathrm{s}$ \\
$\mathrm{T}_{\text {idle-ca/ed }}$ & $192 \mu \mathrm{s}$ \\
$\mathrm{T}_{\text {sleep-idle }}$ & $0.6 \mathrm{~ms}$ \\
$\mathrm{~T}_{\text {idle-sleep }}$ & $192 \mu \mathrm{s}$ \\
$\mathrm{T}_{\text {tx-idle }}$ & $2 \mu \mathrm{s}$ \\
$\mathrm{T}_{\text {idle-tx }}$ & $192 \mu \mathrm{s}$ \\
$\mathrm{T}_{\text {rx-idle }}$ & $2 \mu \mathrm{s}$ \\
$\mathrm{T}_{\text {idle-rx }}$ & $192 \mu \mathrm{s}$ \\
\hline
\end{tabular}

\subsection{Simulation of PEM}

This test is to evaluate the energy consumption tendency of PEM in different states. In the simulation results shown in Figure 3, ARM SA-1100 consumes the energy of $65 \mathrm{~J}$ when running 200 seconds. The trend of PM energy consumption depends on the processes tasks: packet transmission and route maintenance. PM has different energy consumption in different states: the Run one has a majority of energy consumption which determines the energy change trends, the Idle one follows and the SLEEP one is at least. Furthermore, the energy consumption for state transitions is very small, which is almost negligible. Hence, increasing sleep time and lowering power consumption in the state is the main solutions for reducing processor energy consumption.

\subsection{Simulation of TEM}

This experiment is to evaluate the energy consumption tendency of TEM in different states. Figure 4 explores that the system load conditions determines the trends of energy consumption, while the data packet sending has a great impact on the system loads. In this experimental load conditions, TM has the largest energy consumption in the RX state, followed by the TX state. The RX one has more than half of the total energy consumption, having the greatest impact on the energy trend curve. That because, in this experiment, all packets within the sensing area will be received by nodes and the node determines to accept or discard the packet, so that the number of packet receiving is much greater than the number of packets sent. The energy consumption of TM is similar in the states of IDLE, CCA/ED and state transition, and the Sleep one is at least. Therefore, to meet the needs of
WSN applications, TM should adopts multi-hop shortrange wireless communication, so that more nodes can be in the sleep state to reduce power consumption and to decrease the single-hop communication distance.

\subsection{Simulation of SEM}

This experiment compares the energy consumption of $\mathrm{SM}$ in different sensing periods $\left(T_{\mathrm{s}}=5 \mathrm{~s}\right.$ and $\left.10 \mathrm{~s}\right)$. Figure 5 indicates that the SEM energy consumption curve is a straight line, while the slope of the $10 \mathrm{~s}$ line is twice than the $5 \mathrm{~s}$ one, that is, the energy consumption in the $10 \mathrm{~s}$ period is twice than the $5 \mathrm{~s}$ one. Figure 5 further reveals that the energy consumption of SEM is inversely proportional to the sensing period, which has no related to the protocol and load traffics.

\subsection{Evaluation of NEM}

The experiment evaluates the influence of PM, TM and SM to the overall energy consumption of the node, and analyze the node energy consumption in certain circumstances within different locations. This experiment adopts MSP430f149 microprocessor [11], CC2420 communication module, and DS18B20 temperature sensor.

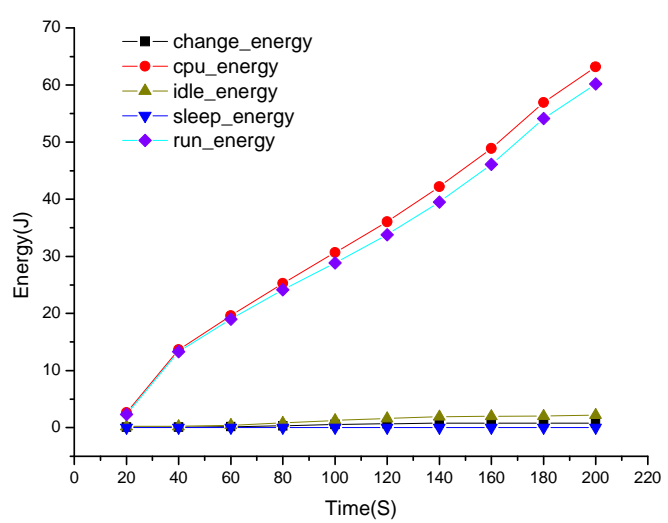

Figure 3. PEM state energy consumption.

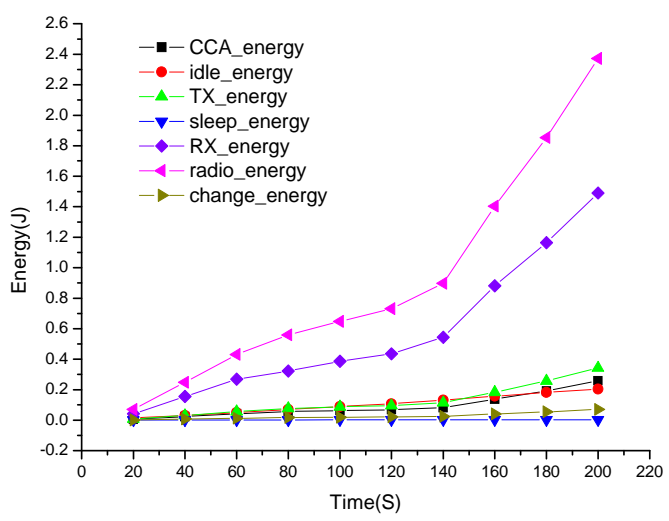

Figure 4. TEM state energy consumption. 


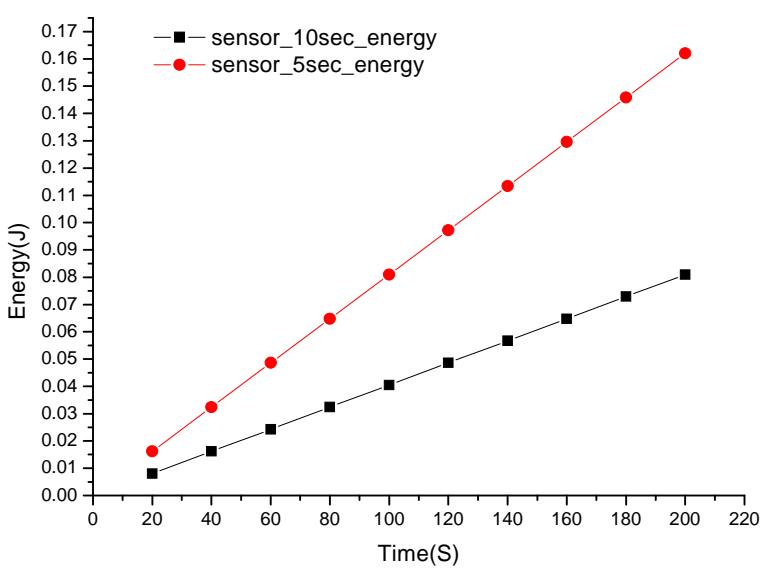

Figure 5. SEM energy consumption.

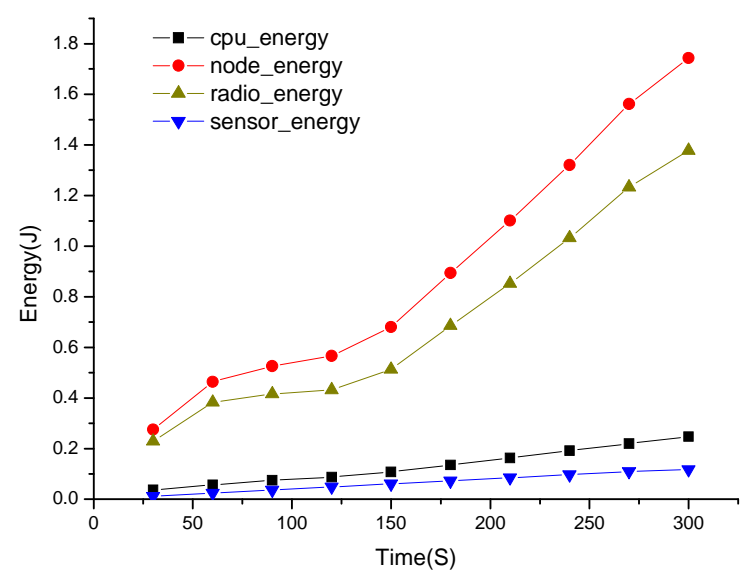

Figure 6. Single node energy consumption in DSR protocol.

This experiment deploys a $1000 \times 500 \mathrm{WSN}$ sensing area within a random distribution of 20 nodes. The WSN nodes employ the routing protocols with DSR [12], the system tasks including sending data packets and maintaining routing protocol. The simulation time length is set to $300 \mathrm{~s}$ : from $0-50 \mathrm{~s}$, data is sent with $3 \mathrm{~s}$ period; from $50-130 \mathrm{~s}$, data is sent by a passion distribution with the expected value of $10 \mathrm{~s}$; from $130 \mathrm{~s}$ to $230 \mathrm{~s}$, data is sent with 1s period; from $230-300 \mathrm{~s}$, data is sent by a passion distribution with the expected value of $5 \mathrm{~s}$. The range of wireless communication is $200 \mathrm{~m}$. The analysis of energy consumption is based on a random selecting node.

Figure 7 shows the energy consumption of node and modules under the DSR protocol, in which TM has the largest energy consumption, followed by PM, and SM has the least consumption. Hence, TM is the main energy unit in a WSN node. In DSR protocol, the energy consumption situation from node 0 to node 19 is shown in Figure 7, in which we can find that the edge nodes consume less energy than the center nodes because the center nodes carry more routing tasks in WSN networks.

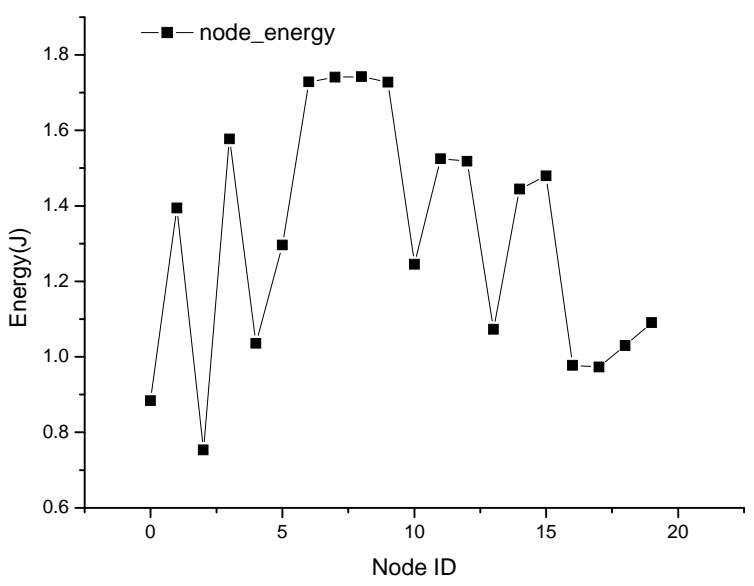

Figure 7. Multi-node energy consumption distribution in DSR protocol.

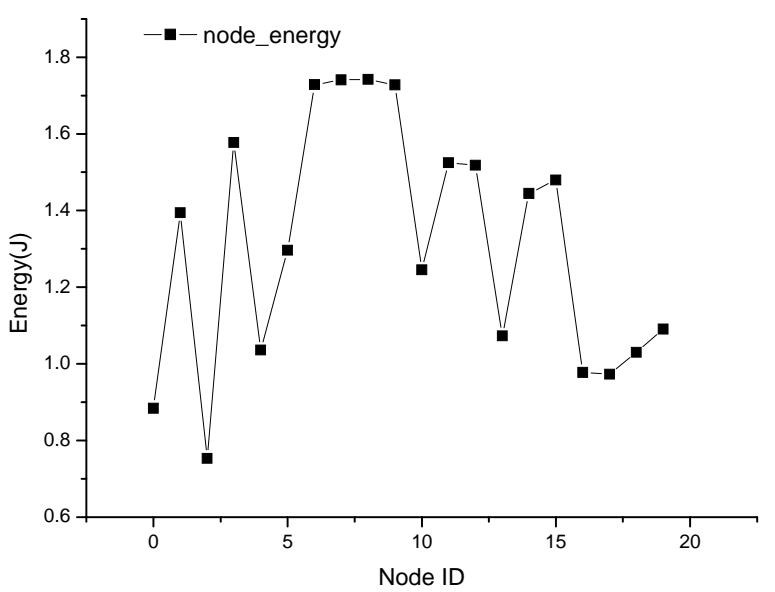

Figure 8. Multi-node energy consumption distribution in DSR protocol.

\section{Conclusion}

Current WSN researches focus more on communication protocols than on energy consumption modeling. Traditional energy analysis method is to deduce the energy consumption statuses of nodes and networks based on the theoretical energy consumption data or theoretical models of system components. Most of existed energy models only analyze the energy status of communication module, being lack of studying the overall energy consumption from the view of nodes. By modeling the energy consumption of different node components in different operation modes and state transitions, this paper proposes a new node energy model based on the eventtrigger mechanism. This model can be used to analyze the energy status of WSN nodes and systems, to evaluate the communication protocols and to help to deploy nodes and construct WSN applications. 


\section{Acknowledgements}

The authors would like to thank all the colleagues and copartners who have contributed to the study. The work was supported by the grants from the Doctoral Fund of Ministry of Education of China (200802131024), the Harbin Science and Technology Development Funds (2009RFLXG009), the National Key Technologies R \& D Program of China (No. 2011BAH04B03), and the International scientific cooperative research program of China (2010DFA14400).

\section{References}

[1] I. F. Akyildiz, W. Su, Y. Sankarasubramaniam and E. Cayirci, "Wireless Sensor Networks: A Survey," Computer Networks, Vol. 38, No. 4, March 2002, pp. 393-422. doi:10.1016/S1389-1286(01)00302-4

[2] I. Howitt and J. Wang, "Energy Efficient Power Control Policies for the Low Rate WPAN," Sensor and Ad Hoc Communications and Networks, Vol. 10, 2004, pp. 49-52.

[3] B. Han, D. Z. Zhang and T. Yang, "Energy Consumption Analysis and Energy Management Strategy for Sensor Node," International Conference on Information and Automation, Proceedings of the 2008 IEEE, Vol. 6, 2008, pp. 211-214.

[4] F. Lu, G. Z. Xu and R. D. Ying, "Power Consumption Simulation Model Based on the Working Status of Intel PXA250 Processor," Control \& Automation, Vol. 21, No. 1, 2005, pp. 131-132.
[5] X. F. Wang, J. Xiang and B. J. Hu, "Evaluation and Improvement of an Energy Model for Wireless Sensor Networks," Chinese Journal of Sensors and Actustors, Vol. 22, No. 9, 2009, pp. 1319-1321.

[6] X. Li, "Network Modeling and Simulation with OPNET Modeler,” Xidian University Press, Xi’an, 2006, pp. 1-15.

[7] Intel Corp., "Intel StrongARM SA-1100 Microprocessor,” Developer's Manual, August 1999.

[8] V. Tsiatsis, S. Zimbeck and M. Srivastava, "Architecural Strategies for Energy Efficient Packet Forwarding in Wireless Sensor Networks," Proceedings of the 2001 International Symposium on Low Power Electronics and Design, Vol. 3, 2001, pp. 25-31. doi:10.1145/383082.383 102

[9] T. H. Jiang and Z. W. Jiang, "Characteristics and Application of the Digital Temperature Sensor DS18B20," Electronic Technology, Vol. 12, 2003, pp. 46-49.

[10] Y. Bin and S. Bin, "Modify AODV for MANET/INTERNET Connection through Multiple Mobile Gateways," Advanced Communication Technology, Vol. 11, No. 3, 2009, pp. 1519-1523.

[11] Q. Yang, X. H. Chen and J. H. Shi, "Low Power Design of the Terminal Node for Wireless Sensor Network," Journal of Xiamen University (Natural Science), Vol. 47, No. 3, 2008, pp. 357-358.

[12] W. Huang, N. Y. Li and Q. Sun, “Accomplishment and Simulation for Dynamic Source Routing (DSR) Protocol Based on OPNET in MANET," Journal of Wuhan University of Technology, Vol. 29, No. 2, 2005, pp. 182-185. 